\title{
Zur Wirkung von Oxytocin auf einen autorhythmischen glatten Gefässmuskel
}

Bei unseren Untersuchungen über autorhythmische Gefässmuskulatur ${ }^{1-4}$ prüften wir die Wirkung strukturverwandter Peptide auf die spontan aktive Rattenpfortader in vitro. Dieses Gefäss zeigt unter isometrischen Bedingungen eine konstante Autorhythmik ${ }^{1,5-7}$ (Figur). $\mathrm{Val}_{5}$-Angiotensin II erhöht die Kontraktionsamplitude konzentrationsabhängig; dieser Effekt kommt durch einen direkten Angriff an der Muskelzelle zustande ${ }^{3}$. Oxytocin führt zu einer Abnahme der Kontraktionsampliture und in einer Konzentration von $5 \times 10^{-7} \mathrm{~g} / \mathrm{ml}$ zu einem Sistieren der Spontanaktivität² (Figur). Die vorliegenden Versuche sollen klären, inwieweit für diese Wirkung adrenerge Mechanismen eine Rolle spielen.

Methodik. Registrierung der isometrischen Spannungsentwicklung (Vorspannung $800 \mathrm{mg}$ ) mit Dehnungsmessstreifen in einer Tyrode-Lösung von $35^{\circ} \mathrm{C}$, Berechnung der mittleren Einzelkontraktion und der Frequenz. Darstellung der Ergebnisse in Prozenten der Ausgangsbedingungen $(\bar{X} \pm S \bar{x})$.

Befunde. Oxytocin senkt die Kontraktionsamplitude in einer Konzentration von $10^{-7} \mathrm{~g} / \mathrm{ml}$ auf $1,52 \pm 0,35 \%$ der Ausgangsbedingungen $(=100 \%)$. Phenoxybenzamin $\left(5 \times 10^{-6} \mathrm{~g} / \mathrm{ml}\right)$ beeinflusst weder für sich die Amplitude $(109,56 \pm 8,18 \%)$ noch den Oxytocin-Effekt $(6,43 \pm$ $1,13 \%)$. Propranolol $\left(5 \times 10^{-6} \mathrm{~g} / \mathrm{ml}\right)$ lässt die Amplitude unverändert $(100,9 \pm 16,09 \%)$ und hemmt nicht die $\mathrm{Ab}$ nahme der Amplitude unter Oxytocin $(1,63 \pm 0,04 \%)$. Eine Vorbehandlung der Tiere mit Reserpin $(0,5 \mathrm{mg} / \mathrm{kg}$ 48,24 und $12 \mathrm{~h}$ vor dem Versuch) beeinflusst die Wirkung von Oxytocin nicht. Unter Iproveratril $\left(10^{-6} \mathrm{~g} / \mathrm{ml}\right)$ nimmt die Amplitude auf $18,88 \pm 2,68 \%$ ab, nach zusätzlicher Gabe von Oxytocin $\left(10^{-7} \mathrm{~g} / \mathrm{ml}\right)$ auf $2,83 \pm 0,6 \%$. Die Wir-

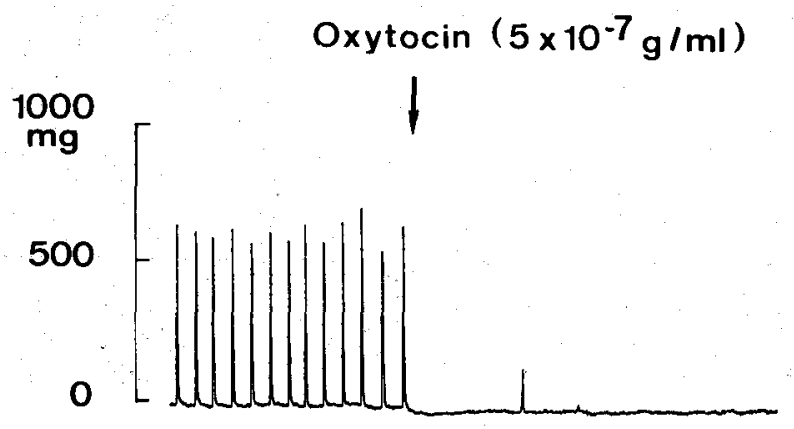

$\min$

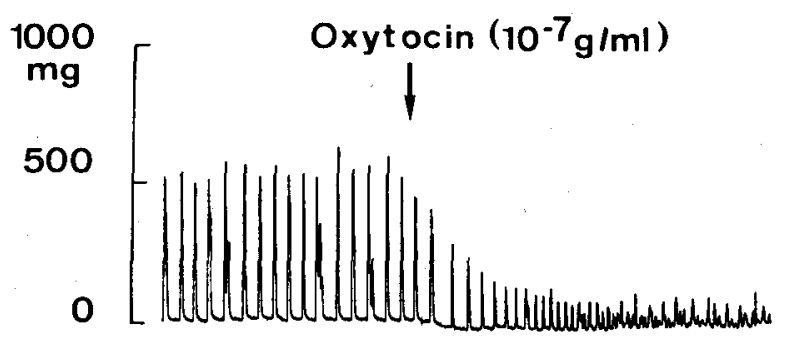

min

Oxytocin führt konzentrationsabhängig zu einer starken Abnahme der Kontraktionsamplitude. kung des Oktapeptides bleibt auch nach mehrfacher Gabe am gleichen Präparat völlig konstant (Tabelle). Eine schwache Konzentration $\left(10^{-8} \mathrm{~g} / \mathrm{ml}\right)$ bleibt auch nach vorheriger Gabe einer starken Konzentration $\left(5 \times 10^{-7} \mathrm{~g} /\right.$ ml) unverändert wirksam. Die Kontraktionsfrequenz wird deutlich geringer beeinflusst, sie nimmt bei Abnahme der Amplitude zu.

Die Befunde zeigen, dass die Wirkung von Oxytocin ohne Vermittlung adrenerger Mechanjsmen zustande kommt und somit durch einen direkten Angriff an der glatten Muskelzelle erklärt werden muss. Eine Tachyphylaxie ist nicht $\mathrm{zu}$ beobachten. Angiotensin II und Oxytocin sind somit zwei an der Rattenpfortader gegensätzlich wirkende Oktapeptide mit anscheinend ähnlichem Angriffsort und damit für Untersuchungen über ihre Wirkungsspezifität an diesem Objekt gut geeignet ${ }^{8}$.

\begin{tabular}{lr}
\hline Ausgangsbedingung & $100 \pm 9,73 \%$ \\
1. Oxytocin $\left(10^{-7} \mathrm{~g} / \mathrm{ml}\right)$ & $2,53 \pm 0,01 \%$ \\
Tyrode-Lösung & $93,4 \pm 5,2 \%$ \\
2. Oxytocin & $2,27 \pm 0,45 \%$ \\
Tyrode-Lösung & $99,96 \pm 12,76 \%$ \\
3. Oxytocin & $3,88 \pm 0,65 \%$ \\
Tyrode-Lösung & $97,16 \pm 10,08 \%$ \\
\hline
\end{tabular}

Die Wirkung von Oxytocin auf die Kontraktionsamplitude bleibt auch nach dreimaliger Gabe am gleichen Gefässpräparat unverändert $(\bar{x} \pm S \bar{x}, n=6)$.

Summary. The influence of oxytocine on the spontaneously contractile portal vein of the rat has been investigated. This octapeptide decreases the active tension and abolished it completely at a concentration of $5 \times 10^{-7} \mathrm{~g} / \mathrm{ml}$. The effect is not caused by an adrenergic mechanism but by a direct action on the smooth muscle cell.

\section{VOTH und W. KäVENHEIM}

Neurochirurgische Klinik der Johannes-GutenbergUniversität, D-6500 Mainz (Deutschland), 26. August 7971.

1 D. Voth, R. Schipp und M. Agsten, Naunyn-Schmiedebergs Arch. Pharmak. exp. Path. 264, 321 (1969).

2 D. Voth, R. Schipp, M. Agsten, K. Schürmann, M. Kohlhardt und J. Dudek, Arch. Kreislaufforsch. 60, 364 (1969).

3 D. Voth, M. Agsten, R. Schipp und H. Lübcke, Arch. Kreislaufforsch. 65, 41 (1971).

4 R. Schipp, D. Voth und I. Schipp, Z. Anat. EntwGesch. 134, 81 (1971).

5 J. Axelsson, B. Wahlström, B. Johannsson und O. Johnsson, Circulation Res. 27, 609 (1967).

6 H. Mislin, Rev. suisse Zool. 76, 1063 (1969).

7 K. H. Booz, Ann. Univ. Sarav. Med. 7, 115 (1959).

8 Mit Unterstützung durch die Deutsche Forschungsgemeinschaft, Bad Godesberg (Nr. Vo 96/3-5). 